\title{
On the Perceived Usefulness of Scaling Techniques in Market Analysis
}

\author{
Michael D. Johnson \\ University of Michigan \\ Elania J. Hudson \\ University of Michigan
}

\begin{abstract}
This study examines the perceived usefulness of alternative spatial and tree-based similarity scaling techniques in a market analysis task. These techniques are typically evaluated on their ability to fit or explain customer input data. The psychological reaction of individuals who use these techniques to analyze markets has been largely ignored. The study reveals that spatial representations are perceived as more useful than tree-based clustering techniques, even though the latter provide a better fit to customer perceptions. The results have implications for both the use and development of scaling techniques in psychology and marketing.
\end{abstract}


The general goal of market research is to provide information for market decisions. Yet research methods and techniques are often evaluated irrespective of the managers who ultimately use them. Researchers typically evaluate new methods on the basis of various statistical criteria. Managers and other users of the research evaluate these methods on the basis of the customer and market insights they provide. Thus any comprehensive evaluation of market research methods should consider both the method's ability to capture or explain some input data and the user's perception of its value.

Perceptual scaling techniques exemplify this problem. Customer perceptions of product or service similarity are typically analyzed with the use of spatial and/or tree-based scaling techniques. Spatial techniques, most notably multidimensional scaling or MDS, depict customer perceptions of brands or product categories as points in a spatial representation or product map. Clustering techniques, such as hierarchical clustering and additive tree scaling, depict stimuli as nodes or branches in a tree- like structure. Market researchers have gained a thorough understanding of these different methods and the trade-offs inherent in their use. Still, little is known of the perceived usefulness of these techniques for the purpose of market analysis. On the one hand, the intuitive appeal of spatial scaling has greatly facilitated its acceptance to the point where it has become a standard tool by which managers analyze product markets and develop market strategies (Green, Carmone, \& Smith, 1989). On the other hand, tree-based techniques are often superior at explaining customer perceptions and performing strategic marketing tasks (O'Shaughnessy, 1984; Srivastava, Leone, \& Shocker, 1981).

The goal of this article is to explore several factors that should affect the perceived usefulness of similarity scaling techniques in a market analysis task. Although a number of studies make observations in this regard, the question has not been systematically studied. Market researchers continue to evaluate scaling techniques primarily on their ability to capture or fit the perceptual data at hand. This tradition fails to consider that managers use the output of these techniques as part of an overall market analysis in order to attain an understanding of customer perceptions, to size up the nature of competition in a market, and to identify potential market opportunities.

Shepard's (1981) conceptualization of the "psychophysical complementarity" of spatial representations is used here to argue that spaces are perceived as more useful than tree-based representations in this task environment. Unlike trees, spatial representations present customer perceptions in a form that complements managers' own perceptual apparatus. Alternatively, tree scaling techniques are arguably more useful than spaces. They provide a market representation that is both more consistent with customers' decision processes and more varied in scope from broad to narrow market definitions. Other hypotheses posit that the perceived usefulness of these techniques should depend on the brand versus category level of the products or services studied and the manager's familiarity with the brands or categories involved. After developing the hypotheses, an empirical study is reported in which subjects used spaces or trees to analyze various markets and then evaluated the usefulness of the representations.

\section{SPATIAL AND TREE-BASED SIMILARITY SCALING}

Researchers utilize two classes of techniques when scaling customer perceptions of the proximity of alternative brands or product categories: spatial scaling and tree scaling or clustering. Spatial scaling using MDS represents products and other stimuli as varying on a small number of continuous dimensions in some underlying geometric space (Kruskal, 1964; Shepard, 1962). Perceptual distance or proximity is modeled as the distance, typically Euclidean, between pairs of products in the space. Tree-based scaling techniques and clustering schemes, from S. C. Johnson's (1967) hierarchical clustering schemes (HCS) to additive and extended tree scaling (Corter \& Tversky, 1986; Sattath \& Tversky, 1977), represent products as external nodes in a tree structure. Perceptual distance in a tree is proportional to the length of the paths separating the products.

To illustrate, both ultrametric trees produced using HCS (average linkage method) and MDS solutions (Euclidean metric in two dimensions) are presented in Figures 1 and 2, respectively. Each figure provides a solution for a set of category-level stimuli (vehicle types) and brand-level stimuli (sedans). The 
input data for these solutions were average pairwise similarity judgments collected during Phase 1 of the empirical study described later. The tree for vehicles separates cars from trucks, and sports cars from both. The car branch separates sedans from wagons, and the truck branch separates vans, pickups, and sport utility vehicles (SUVs). The tree for sedans separates subcompacts (Escort and Civic) from the larger sedans, and then separates large American sedans from midsize sedans. Among the midsize sedans, the two General Motors sedans are grouped separately from a core class of popular models (Accord, Camry, Maxima, and Taurus). Meanwhile, the MDS solution for vehicles separates the options by size and function (sporty vehicles lower left, practical vehicles upper left, and larger utility vehicles to the right). The MDS solution for sedans also shows a clear size dimension from left to right and what appears to be a style dimension running from top to bottom. Unlike the tree, the spatial solution for sedans has the GM midsize sedans (Grand Am and Corsica) positioned in the midst of Japanese competitors.

Both types of techniques have proven useful to researchers and managers interested in analyzing product markets. Market analysis, in this context, involves understanding customers’ perceptions, analyzing the nature of competition, and identifying market opportunities.

An increasingly sophisticated number of algorithms for analyzing proximity data with the use of both spatial and tree-based techniques have emerged over the years to handle problems ranging from individual differences, to asymmetric data, to uncertainty (DeSarbo, Johnson, Manrai, Manrai, \& Edwards 1992; DeSarbo \& Rao, 1984; MacKay \& Zinnes, 1986). There are also hybrid techniques, which simultaneously represent proximities with the use of both types of structures (Carroll, 1976; Shepard, 1980). However, of primary interest in this research is the perceived usefulness of the two general classes of scaling techniques, spaces and trees, that are commonly available to managers. Managers use these scaling techniques to analyze customer perceptions of competitors and identify market opportunities. The focus, therefore, is on the basic spatial and tree scaling techniques illustrated in Figures 1 and 2.

\section{Psychophysical Complementarity and Market Analysis}

One approach is to view perceived usefulness as a function of the degree to which the output of a market research technique complements an individual's own perceptual apparatus. Here spatial representations have a unique advantage over trees. MDS solutions have always been seen as quite intuitive to managers (Doyle, 1971; Green, 1975). In this research, the conception of distance 

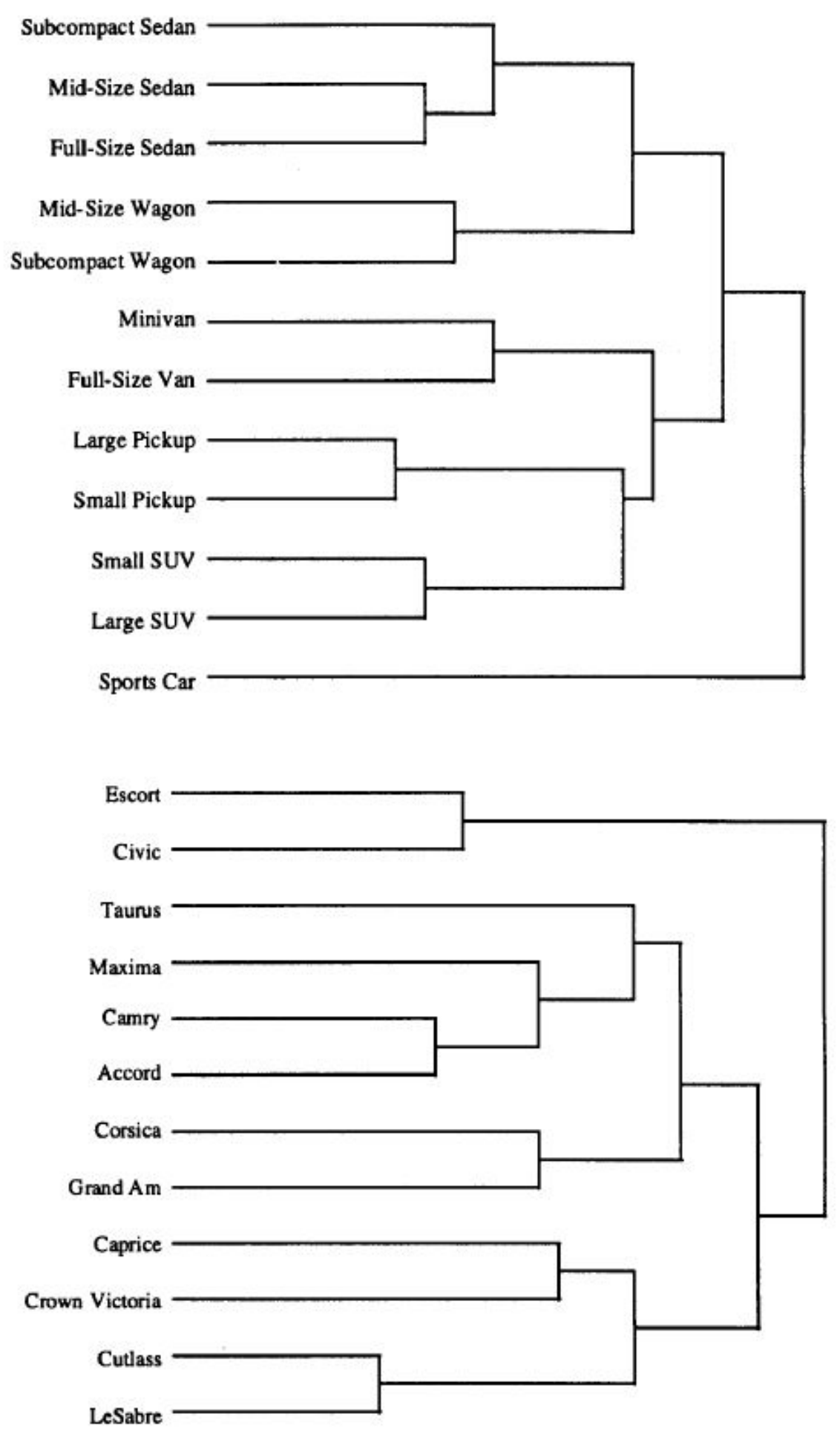

Figure 1 Hierarchical clustering schemes for vehicles and four-door sedans. 

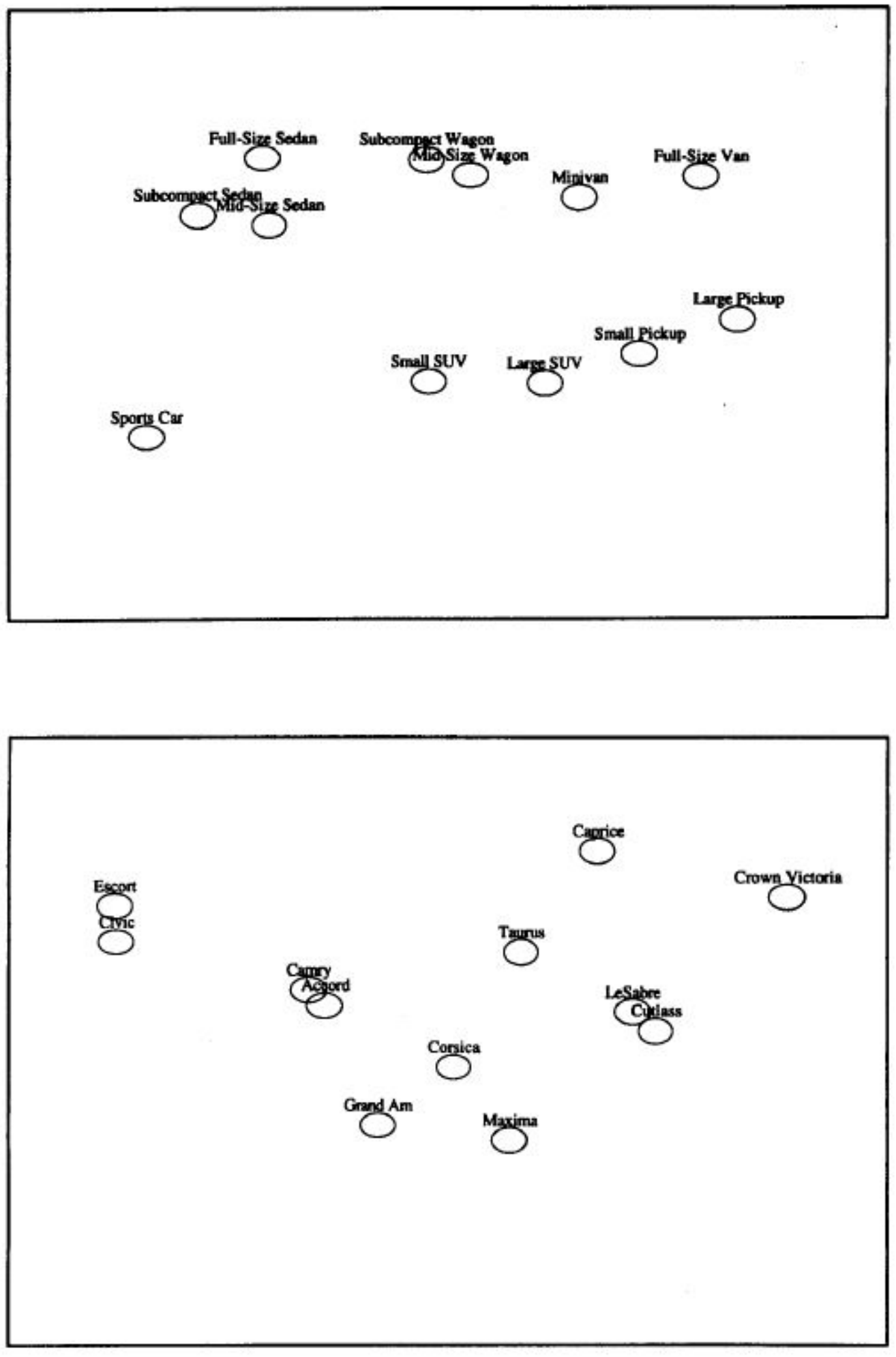

Figure 2 MDS solutions for vehicles and four-door sedans. 
"as the crow flies," or Euclidean distance, provides a straightforward basis for interpreting spatial solutions. Relating psychological distances among stimuli to the lengths of the paths separating nodes in a tree is less intuitive.

In his introduction to the theoretical foundations of multidimensional scaling, Shepard (1972) argued that the value of spatial scaling techniques like MDS lies in their ability (a) to uncover the hidden pat- tern or structure of perceptions, and (b) to represent this structure in a form that is much more accessible to the human eye-as a picture or map. Ten years later, Shepard revisited this issue of psychophysical complementarity when he argued that

Because our commerce with the world necessarily takes place through the spatially very different medium of a two-dimensional boundary, internal representations need not literally resemble their corresponding external objects and events. The internal and the external must nevertheless approximate a kind of complementary mesh. (Shepard, 1981, p. 279)

From an evolutionary perspective, the complementarity that exists be- tween internal relations (perceptions) and external stimuli (such as product spaces) has basic survival value. This is not to say that internal and external representations are similar in the sense that they resemble each other; the internal representation and its object are confined to very different domains. The isomorphism between internal and external representations is more of one in which internal representations, or how people perceive the world, complement or mesh with the external environment.

A large part of market analysis involves gaining an understanding of the market as a whole, or the proverbial "forest for the trees." Shepard's psychophysical complementarity argument suggests that spatial representations are perceived as more useful when performing such a task. Perceptually, managers should comprehend and interpret spatial representations with relatively little trouble. Unlike trees, spatial representations highlight the primary dimensions underlying the market as a whole and, hence, the nature of competition. This gestalt, or "forest view," should also allow managers to quickly identify poten-tial new product opportunities.

Anecdotal support for the intuitive superiority of spaces exists in the language managers use to analyze markets for strategy development. Products are positioned (in spaces) rather than branched (onto trees). Likewise, managers strive to identify unique product niches in what is clearly a spatial metaphor. Although focusing on a different task, M. D. Johnson and Horne (1992) support a similar type of effect. They had consumers indicate their perception of a particular product or service directly in existing spatial or tree-based scaling solutions. The consumers found it significantly easier to locate a product or service as a new point in a space rather than a new branch on a tree. Shepard's psychophysical complementarity argument provides the basis for Hypothesis 1:

H1: Spatial scaling solutions are generally perceived as more useful than tree scaling solutions in a market analysis task.

Unfortunately, this prediction has been largely assumed rather than empirically tested. There are also a number of compelling arguments in favor of an alternative hypothesis. One is that tree-based solutions, specifically hierarchical clustering schemes, provide a representation of a market that is more consistent with customers' own hierarchical decision processes (Bauer \& Herrmann, 1995; Srivastava et al., 1981). Customer decision making often occurs in an elimination-type or hierarchical fashion. The different branches in a tree may be viewed as choice points in this hierarchical decision process (Hauser, 1986; Tversky \& Sattath, 1979). A second argument is that hierarchical trees offer unique strategic benefits to managers. They are superior at delineating a market's competitive structure, from the nature of com- petition among specific brands to the broader, more categorical competition that exists among product types (O'Shaughnessy, 1984). A third argument is that certain tree scaling techniques, such as the Tversky and Sattath (1979) ADDTREE program, are able to reveal important differences in the extent to which particular products or services are representative or prototypical members of a category 
(Hodgkinson, Padmore, \& Tomes, 1991). These prototypical offerings serve as cognitive reference points against which other products tend to be compared.

Yet perhaps the best argument for the perceived superiority of trees over spaces in a market analysis task is based on their difference in fit. Scaling fit is the degree to which the fitted distances between stimuli in a spatial or tree structure match or explain the input data (such as perceived similarity or relative preference). Research studies that have compared these methods in the scaling of conceptual stimuli reveal that trees typically dominate spaces with respect to fit (M. D.Johnson \& Fornell, 1987; M. D. Johnson, Lehmann, Fornell, \& Horne, 1992; Pruzansky, Tversky, \& Carroll, 1982). This objective difference in statistical fit may mediate managers' perceptions of the use value of scaling solutions. Other things being equal, if a market representation is put in the hands of a manager for the purpose of market analysis, the manager should perceive the technique as more useful in completing the task as fit improves. Outputs that provide better fits likely contain more information regarding customer perceptions. This information should help the manager to make better decisions. Taken together, these arguments suggest an alternative to Hypothesis 1 whereby tree scaling solutions may be perceived as more useful than spatial scaling solutions.

\section{The Level of Competition: Brands versus Categories}

If the statistical fit of a scaling solution mediates its perception of usefulness, then it will be critically important to examine the level of competition involved. This is because the statistical fit of spatial versus tree scaling outputs varies with the level of competition. At one level, managers analyze very direct competition among brands from the same category (e.g., cereals, fast-food restaurants). At another level, managers analyze the competition that exists across categories themselves (e.g., breakfast foods, dining alternatives). The major difference between these two levels of competition from the customer's perspective is the level of abstraction involved (M. D. Johnson, 1984). At the brand level, customers' judgments and choice processes are based on more concrete product descriptions or representations. Categories are commonly judged and evaluated with the use of more general or abstract representations. Although, for example, vehicles may be compared on such abstract attributes as practicality and fun, specific variants on four-door sedans may vary on the presence or absence of such attributes as a CD player, a passenger side airbag, and antilock brakes.

Recent research on customer perceptions demonstrates how the fit of spatial and tree scaling techniques varies between these two levels. Three separate studies, one in Germany (Herrmann, 1992) and two in the U. S. (M. D. Johnson \& Fornell, 1987; Johnson et al., 1992), show that the fit of spatial scaling improves relative to tree scaling when category-level stimuli rather than brands are analyzed. M. D. Johnson et al. (1992) tested alternative explanations for this effect and found that it is likely the result of a fundamental difference between space and tree scaling. Spatial scaling with the use of MDS is conceptually and analytically similar to factor analysis and principal components (Shepard, 1972). MDS is very much a data-reduction technique in that it describes differences among products on a small number of latent, abstract, or underlying dimensions (e.g., sportiness or style in the solutions in Figure 2). Clustering techniques and tree scaling are less reductionist in nature. Rather, they reveal salient distinctions or attributes of perception (Sattath \& Tversky, 1977). In short, the observed differences in fit are driven by the inherent concreteness or abstractness of the product perceptions being scaled. If the fit of a scaling solution mediates perceived usefulness, these results have important implications for managers who analyze markets at the brand and category levels. When analyzing brands as opposed to categories, managers may find tree-based scaling solutions increasingly useful. When analyzing the more abstract categories, the relative usefulness of latent spatial scaling solutions should improve. This prediction is stated as Hypothesis 2:

H2: The perceived usefulness of spatial scaling solutions increases relative to that of tree scaling solutions when analyzing product categories as opposed to brands. 
Hypotheses 1 and 2 are not mutually exclusive. Spatial solutions could dominate for both brands and categories (Hl), but less so for brands than for categories (H2). In the study described below, subjects use either spaces or trees to analyze sets of categories and sets of brands. The subject's tasks in each case are to analyze the nature of the customer perceptions and the nature of competition in the market, and to identify a potential new product opportunity. The dependent variables are a battery of questions that tap the perceived usefulness of the tree (space) in performing the tasks. The objective quality of the analyses and decisions made using the spaces and trees is beyond the scope of the study. In effect, Hypothesis 2 predicts an interaction effect involving the type of scaling solution (space versus tree) and the type of stimuli being analyzed (brands versus categories) on perceived usefulness. This usefulness should increase for spaces relative to trees when brands rather than categories are being analyzed.

On the surface, Hypothesis 2 may appear counterintuitive to researchers studying differences between perceptual (imagined) stimuli and conceptual (semantic) stimuli. This research shows that spatial representations provide a better fit when scaling perceptual stimuli (such as tones or colors), whereas treebased representations provide a better fit when scaling conceptual or semantic stimuli (such as occupations; Pruzansky et al., 1982; Tversky \& Hutchinson, 1986). Perceptual stimuli are more concrete and easier to visualize and encode than are conceptual or semantic stimuli (Anderson, 1983). Similarly, brands are more concrete and easier to visualize than are product categories. By inference, it may be argued that spatial representations become more useful when analyzing brands rather than categories.

However, the distinction made here between brands and categories is entirely within the domain of conceptual or semantic stimuli. Thus brand-level product concepts cannot be directly equated with perceptual stimuli such as colors or tones (M. D. Johnson \& Fornell, 1987). Hypothesis 2 is driven by inherent differences in abstraction that underlie perceptions of concrete brand concepts versus more abstract product category concepts. The hypothesis is not based on differences in customers' ability to visualize and encode perceptual versus conceptual stimuli. The two streams of research are, in fact, quite consistent in their findings. They both show that the fit of tree-based scaling is generally superior to spatial scaling within the domain of conceptual or semantic stimuli. The empirical study (described below) again finds that tree-based scaling techniques provide better-fitting solutions for both brands and categories. At the same time, the relative fit of spaces increases, and the relative fit of trees decreases, on average, when scaling categories as opposed to brands. This is consistent with Hypothesis 2.

A main-effect difference between brands and product categories is expected with respect to usefulness. Managers are likely to understand customer perceptions of category-level stimuli without the aid of a scaling solution. This assumption rests on well-documented support in the psychology and consumer-behavior literatures showing a particularly salient or basic level of categorization at which categories are most differentiated from one another (Alba \& Hutchinson, 1987). Stimuli from the same basic category exhibit a singularly high degree of inclusiveness or similarity of their members. These basic level distinctions pervade perceptions of category level stimuli and are relatively common within a particular culture (Rosch, 1977). Thus managers should have a good understanding of customer perceptions at this level without the aid of a scaling solution. This is not the case for brands where the distinctions among stimuli are more subtle and idiosyncratic to a particular market segment. It is thus expected that a scaling solution should be of greater use to a manager whose task is to analyze customer perceptions of brands. This prediction is stated formally as Hypothesis 3:

H3: The usefulness of both spatial and tree scaling solutions is greater when analyzing brands as opposed to product categories.

\section{Stimulus Familiarity}

In the empirical study, an attempt was made to hold the managers' familiarity with the different techniques constant by using undergraduate business school students as subjects. These students were participating in a marketing management class and surveyed at a point in the semester at which they had developed a good understanding of market analysis through the use of case studies and lectures. At the 
same time, they had not spent weeks, months, or years using a particular perceptual scaling tool to perform this task, such as product maps. Thus, their familiarity with the different techniques is held relatively constant.

However, it is also important to consider the managers' familiarity with the stimuli in the study. Managers require some base level of familiarity with the objective characteristics of the stimuli whose customer perceptions they are analyzing, such as the general sugar content of Fruit Loops or the relative size of a Camry sedan. Scaling solutions leverage the managers' own knowledge of these stimuli when analyzing a market. Put differently, stimulus familiarity is a prerequisite for using a scaling solution to perform a market analysis task. Thus, the more familiar a subject is with the products in the space or tree, the more useful the technique is in performing the market analysis, which is stated as Hypothesis 4:

H4: The usefulness of both spatial and tree scaling solutions increases with a subject's familiarity with the products or services being analyzed.

The next section details an empirical study that was conducted to test the research hypotheses.

\section{EMPIRICAL STUDY}

The empirical study was conducted in two phases. In Phase 1, subjects were asked to provide pairwise similarity judgments for one of six stimulus sets, each of which contained 12 individual products or services. The sets were chosen to represent a variety of brands and categories where the brand-level stimuli are nested under the category-level stimuli. Presented in Table 1, the sets include major durables (vehicles and sedans), nondurables (breakfast foods and cold cereals), and services (dining-out alternatives and fast-food restaurants). The brand-level stimulus sets were constructed with the use of Simmons market share data. The category-level sets were constructed so as to cross traditional product and service category boundaries and represent a wider range of competition than exists among the brands.

Subjects were recruited from undergraduate business classes at a midwestern university and asked to complete a single questionnaire involving one of the six stimulus sets for course credit $(n=135)$. The subjects were introduced to the entire range of stimuli in the set and the proximity scale to be used. They then rated all 66 product or service pairs on a scale from 1 (very similar) to 7 (very dissimilar). The sample sizes by stimulus set were 18, 24, 24, 24, 23, and 21 for sedans, vehicles, fast food, dining alternatives, cereals, and breakfast foods, respectively. Half the subjects rated the pairs within a set in random order and the other half rated the same pairs in reverse order. Although this information was not used in the study, the subjects then rated the stimuli with respect to their confidence in judging the stimuli, their prototypicality, preference, knowledge, and experience.

The average proximity judgments were then used to develop spatial and tree-based scaling solutions for Phase 2. Two variations on MDS and tree scaling were used so that the results are not an artifact of a particular metric or scaling algorithm. Two-dimensional MDS solutions were developed with the use of the Roskam and Lingoes (1970) SSA analysis under the two most common metrics, Euclidean and city block (Minkowski $\mathrm{r}=2$ and 1, respectively). Tree-based solutions were developed with the use of S. C. Johnson's (1967) HCS with an average linkage method. The Sattath and Tversky (1977) ADDTREE program was also used. Hierarchical clustering with HCS (see Figure 1) produces ultrametric trees that constrain all intracluster distances to be smaller than all intercluster distances, and all intercluster distances for any given pair of clusters must be equal. Although HCS is more common than ADDTREE, the latter is less constrained and provides better-fitting solutions (Sattath \& Tversky, 1977). A resulting advantage of ADDTREE (described earlier) is that it is better able to identify highly representative or prototypical stimuli within a set. The more representative a stimulus is, the closer it is to the root of the tree (i.e., the stimulus is closer to other stimuli in the set than those other stimuli are to each other). Although it provides an alternative to HCS, ADDTREE also uses approximately the same number of independent parameters as the two-dimensional MDS solutions. 
Table 1. Stimulus Sets.

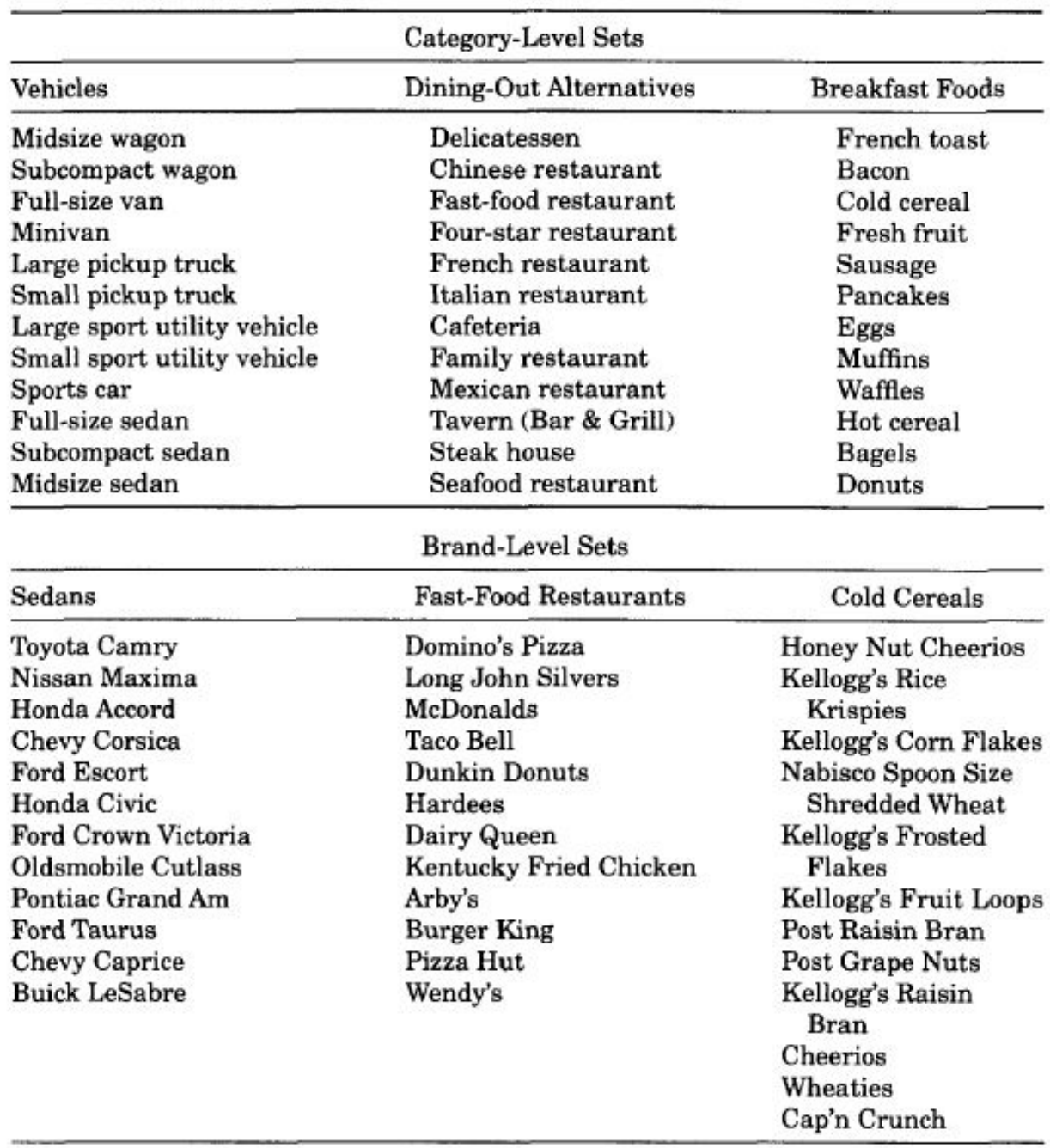

The choice of scaling techniques resulted in four alternative scaling solutions, two trees and two spaces, for each of the six stimulus sets. These 24 solutions were used as input to Phase 2 . The tree and space representations in Figures 1 and 2 are the HCS and Euclidean MDS solutions for the vehicle and sedan data. None of the clusters or dimensions in the trees or spaces were labeled, as this was part of the subjects' market analysis task. A total of 58 subjects from the same population as Phase 1 were recruited for Phase 2, again for course credit. The type of representation (Euclidean space, city block space, HCS, and ADDTREE) was used as a between-subjects variable so as to minimize confusion in having to explain multiple types of representations. Following M. D. Johnson and Horne (1992), the subjects were given an intuitive description of the spaces (maps) or trees they were about to use in which perceptual distance was explained.

Each subject then completed three tasks for each of the six stimulus sets. Task one was to analyze and explain briefly, in writing, the important attributes, dimensions, or features that explain the customers' perceptions of the alternatives in the map (tree). Task two was to analyze and again explain briefly, in writing, the nature of the competition among the alternatives. That is, which alternatives compete most directly as substitutes and which have relatively unique positions. Task three was to use the 
representation as a guide to develop an idea for a new product or service in the category. Subjects were instructed to generate and briefly explain the idea in writing and not to evaluate its potential for success.

Following each market (stimulus set) analysis, the subject responded to a series of Likert-scale questions on a scale from 1 (strongly disagree) to 7 (strongly agree). The statements included:

1. The map (tree) in this case was useful in helping me understand the consumers' perceptions. 2. The map (tree) in this case was useful in helping me assess the nature of the competition among the alternatives.

3. The map (tree) in this case was useful in helping me to generate a new product idea for this category.

4. Overall, the map (tree) was useful in helping me to perform these tasks.

5. I found it easy to think in terms of the map (tree).

6. I agreed with the distances among alternatives in the map (tree).

7. I found the tasks to be confusing.

8. I found the tasks to be easy.

9. I found the tasks to be fun.

10. I am knowledgeable of the alternatives in this case.

11. I am confident evaluating the alternatives in this case.

12. I frequently purchase, consume, or utilize the alternatives in this case.

Questions 1-4 tap the perceived usefulness of the representation in each of the three sub-tasks and overall; 5-9 are scale items used by M. D. Johnson and Horne (1992) in a direct perceptual measurement task utilizing similar spatial and tree-based scaling solutions. Questions 10-12 tap the subjects' relative familiarity with the stimuli in each set.

In every case, the three category-level stimulus sets were presented prior to the three brand-level sets. Because the brand-level stimuli are nested under the category-level stimuli, it was felt that the brands should follow the categories so as not to bias the category-level analyses toward a particular category in the set. The order conditions in the study relate only to order within the sets of brands and within the sets of categories. Accordingly, the subjects were randomly assigned to one of three order conditions, .ABC, BCA, or CAB, for nested category/brand sets A (vehicles/sedans), B (dining-out alternatives/fast-food restaurants), and C (breakfast foods/cold cereals). Under condition ABC, for example, subjects analyzed the stimulus sets in the following order: vehicles, dining-out alternatives, breakfast foods, sedans, fastfood restaurants, and cold cereals. The end result is a 2 X 2 X 3 experimental design involving two types of representations (space versus tree), two stimulus levels (categories versus brands), and three levels of order. There are also nested effect variables for the two types of spatial representations (Euclidean and city block), two types of trees (HCS and ADDTREE), three types of categories (vehicles, dining alternatives, and breakfast foods), and three types of brands (sedans, fast-food restaurants, and cold cereals). A total of 55 out of the 58 subjects completed the tasks as instructed, and these subjects served as the basis for all further scale construction and hypothesis testing.

\section{Usefulness and Familiarity Measures, Analysis, and Manipulation Check}

The Likert questions were used to construct both a usefulness scale as our dependent measure and a familiarity scale as an independent variable in our analysis. Principal-components analysis was used to see which of items 1-9 might be used to form a measure of perceived usefulness. A scale was subsequently constructed with the use of an equally weighted average of Questions 1-5, 7, and 8. Cronbach's coefficient alpha was 0.94 , which supports the reliability of the perceived usefulness scale. Questions 10-12 were also averaged into a reliable stimulus familiarity scale (Cronbach's alpha of 0.93). It is quite possible, given the stimuli, that there are differences in familiarity across our stimulus sets. The familiarity scale was thus standardized within each stimulus set to avoid any confound between stimulus familiarity and the other independent variables. 
An analysis-of-variance model was used to test the hypotheses. The dependent variable was the perceived usefulness scale, and the independent variables were the two-level type of representation factor (spaces versus trees), the two-level stimulus-level factor (categories versus brands), the stimulus familiarity scale as a covariate, the two and three-way interactions involving these factors, the two-level nested variables for the two types of trees and two types of spaces, the three-level nested variables for the three types of brands and three types of categories, and a three-level factor for the order condition. Hypothesis 1 predicts a main-effect increase in usefulness from trees to spaces following Shepard's notion of the psychophysical complementarity of spaces. Hypothesis 2 predicts a type of representation by stimulus-level interaction effect, whereby the perceived usefulness of spaces increases relative to trees when analyzing categories rather than brands. Hypothesis 3 predicts a main-effect increase in usefulness from categories to brands based on managers' a priori knowledge of basic level category differences. Hypothesis 4 predicts a main effect increase in usefulness with stimulus familiarity, as this familiarity leverages the value of the scaling solutions.

The predicted interaction presumes that the fit of spatial scaling improves relative to tree scaling (or decreases less) from the analysis of brands to the analysis of product categories. Following previous studies (M. D. Johnson \& Fornell, 1987; M. D. Johnson et al., 19921, the Euclidean MDS and ADDTREE solutions were examined using Kruskal's monotonic stress measure to check this assumption. As alluded to earlier, average MDS stress decreases (fit increases) from brands (0.115) to categories (0.106), whereas average ADDTREE stress increases (fit decreases) from brands (0.053) to categories (0.071). Although the assumption holds overall, the differences vary by nested brand -category stimulus combination. For example, although the assumption holds well for sedans versus vehicles and for fastfood restaurants versus dining-out alternatives, it does not hold for cold cereals versus breakfast foods. 2 Two types of analyses were conducted in response. As the assumption is more appropriate in some nested combinations than others, the hypotheses were tested across as well as within these combinations. Analyses were also conducted in which Kruskal's stress is used as an independent variable to explain perceived usefulness.

\section{Results}

The results reveal a main effect for type of representation on usefulness; $\mathrm{F}(1,311)=13.767, p<.001$. The subjects rated the spaces as more useful than the trees (average usefulness scale values of 4.558 and 3.969), which supports Hypothesis 1 and Shepard's notion of psychophysical complementarity. It is also consistent with Green's (1975) observations regarding the value of spaces as an intuitive management tool. This result is illustrated in Figure 3, where the perceived usefulness scale rating is graphed as a function of the type of representation and stimulus level. Spaces dominate trees for both brands and categories alike. This is in direct contrast to the arguments presented for the alternative hypothesis, whereby trees would dominate spaces because of their consistency with consumer decision processes, because of their superior insights into broad-based versus more narrow levels of competition, and because of their superior fit (see Footnote 2). Hypothesis 2 was not supported. Recall that this hypothesis predicts a type of representation by stimulus-level interaction effect that did not approach significance. The other two- and the three-way interaction effects were also not significant.

The main effect for stimulus levels predicted under Hypothesis 3 was supported; $F(1,311)=$ 5.802, $p<.05$. As illustrated in Figure 3, the subjects found the trees and spaces more useful when analyzing brands rather than categories. This result is consistent with the notion that managers are more likely to understand customers' category-level perceptions without the aid of a perceptual 


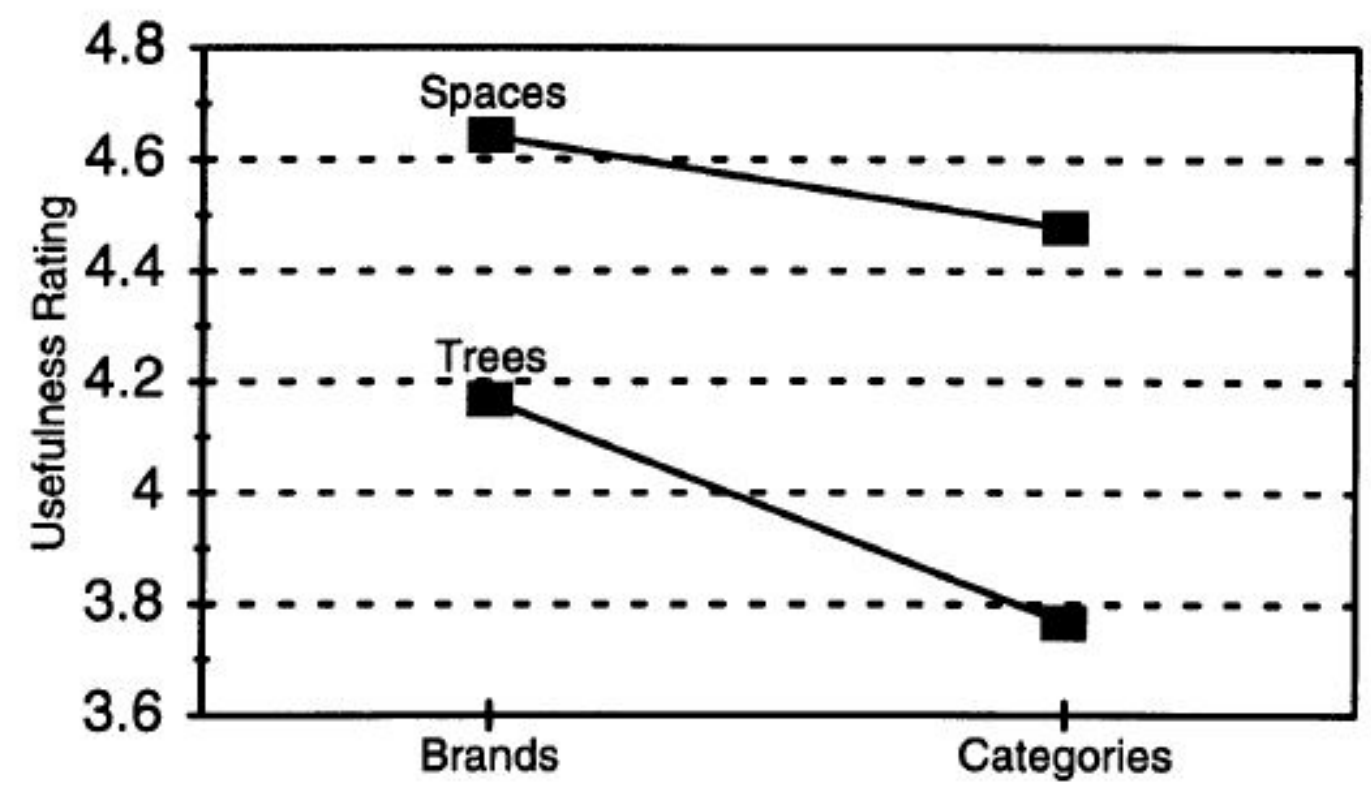

Figure 3 Perceived usefulness by space versus tree and brand versus category conditions.

scaling technique. There was also a large positive effect of stimulus familiarity on perceived usefulness $(F[1,3111]=90.647, \mathrm{p}<.001)$ supporting Hypothesis 4 . The more familiar the subjects were with the stimuli, the more useful they found the spatial and tree-based representations (the correlation between the usefulness and familiarity scales was 0.491). This suggests that stimulus familiarity is an important issue not only for customers themselves but also for the managers who use customer perceptions to analyze markets.

The only other significant effect was a nested effect for the different brand-level stimuli; $F(2,311)$ $=9.779, \mathrm{p}<.001$. The subjects found the spaces and trees more useful for analyzing the cereals (4.468) and the fast-food restaurants (4.777) than for analyzing the sedans (3.865). Recall that stimulus familiarity was standardized within stimulus sets. Unstandardized familiarity was, however, lower for the sedans compared to cereals and fast-food restaurants (scale values of 3.485, 5.297, and 5.515 respectively). The observed nested effect is thus consistent with Hypothesis 4.

The lack of a nested difference in usefulness between the HCS and ADDTREE solutions is also important. Recall that one reason ADDTREE solutions might prove more useful is that they are superior at revealing highly representative or prototypical stimuli. If this was the case, we would have expected the ADDTREE solutions to be rated as more useful than the HCS solutions (that presume ultrametric trees), which they were not.

As previously mentioned, two of the nested stimulus combinations (sedans versus vehicles and fast-food versus dining-out alternatives) provided a better manipulation for the testing of Hypothesis 2 . Even when analyzed separately, however, the type of representation by stimulus-level interaction did not approach significance in any of the three nested combinations. As a further analysis, Kruskal's stress was used as an independent variable to test for possible relationships to perceived usefulness. These analyses were conducted separately for each possible combination of space versus tree and brand versus category condition (because stress levels vary by both stimulus level and type of representation). The usefulness scale was used as the dependent variable and regressed against stress level, the familiarity scale, and their interaction. Only in the case of using treebased representations to analyze brand-level stimuli was there a significant effect, where higher-stress solutions were considered less useful $(\mathrm{t}=-2.758, \mathrm{p}<.01)$. Overall, however, there is relatively little evidence to suggest that the fit of scaling solutions mediates perceived usefulness. 


\section{TASK-SPECIFIC ANALYSES AND RESULTS}

The analyses to this point have treated the three tasks that each subject completed for each stimulus set as a whole. Although the scale measures support the use of one overall usefulness scale, a final analysis was conducted with usefulness questions 1 (usefulness of the map or tree in understanding customer perceptions), 2 (usefulness of the map or tree in analyzing the nature of competition), and 3 (usefulness of the map or tree in generating a new product idea) separately. On the one hand, the overall usefulness scale is a more comprehensive and better measure of the usefulness construct. The multiitem scale reduces error variance in the measure and provides a better test of our hypotheses. On the other hand, some of the arguments in this research suggest that trees are better suited to particular tasks. For example, trees may fare better when subjects are analyzing the nature of competition or the basis of customer perceptions rather than when generating a new product idea. The ANOVA model was estimated three more times, with each individual task measure used as the dependent variable.

The results show that for each task there was a significant main effect for type of representation on usefulness, whereby the spaces were judged as more useful than the trees, consistent with Hypothesis 1. Hypothesis 4, involving stimulus familiarity, was also significant for each individual task measure. The only difference between these analyses and that involving the overall usefulness scale involves Hypothesis 2, where we found that the scaling solutions were generally more useful for the analysis of brands than for product categories. This effect is driven primarily by the competitive analysis task where the effect was significant; $\mathrm{F}(1,330)=6.667, \mathrm{p}<.01$. The effect was not significant in the other two tasks. Finally, Hypothesis 4 was supported in all three tasks where there was a significant effect for familiarity on the usefulness of the scaling solutions.

\section{DISCUSSION}

Both spatial and tree-based scaling solutions offer insights into product perceptions and market structure. This study examined the perceived usefulness of these scaling solutions when performing a marketanalysis task. Subjects used either spaces or trees to analyze both brands and product categories. An important finding of this research is that spatial representations dominate tree-based representations on perceived usefulness. This is in direct contrast to the observation that trees dominate spaces on more statistical criteria such as fit. The results of this study provide empirical support for Green's (1975; Green et al., 1989) assertion that spaces are an intuitive management tool as well as Shepard's $(1972,1981)$ arguments regarding the psychophysical complementarity of geometric spaces.

The results reveal that both types of scaling solutions are perceived as more useful in the analysis of brands rather than product categories. Marketers strive to define and understand competition on a broad level to avoid being myopic (Levitt, 1960). It is interesting and important that the highly valued practices of perceptual mapping and clustering lose some perceived usefulness when analyzing higher level, or more categorical, competition. A third result is that stimulus familiarity leverages the usefulness of both spatial and tree-based solutions. This relatively intuitive finding underscores the need to examine stimulus familiarity from at least two perspectives, that of the customers who provide the input data and that of the managers who use the scaling solutions to analyze a market. Managerial familiarity with brands and categories appears to be a precondition for perceived usefulness.

The results are limited to the particular market analysis tasks employed in the study. There are other management tasks that employ scaling techniques for which trees may be perceived as more useful than spaces. One is when the emphasis is specifically on understanding the more concrete features as opposed to the latent or abstract benefits that drive perception, where feature trees may have a distinct advantage. Another is when the goal is to identify the most representative or prototypical stimuli in a set that serve as cognitive reference points for customers. Here ADDTREE-type solutions may have a distinct advantage. Although no differences were observed between the ADDTREE and HCS solutions, subjects were not asked to identify prototypical alternatives from among the brands or categories. Finally, 
the task focused on clustering brands and categories, not customers. It is important to keep in mind that clustering techniques have proved particularly useful to managers when the goal is to develop a customeror market-segmentation scheme (Kinnear \& Taylor, 1996).

A potential limitation of the empirical study is that subjects were required to analyze categories prior to analyzing brands. By design, this kept the subjects from focusing on particular categories in the category-level analysis tasks. This potential confound may explain the observed main effect where the scaling solutions were more useful when analyzing brands. There may have been a learning effect in using both the spaces and trees that favored the brand-level stimuli. An analysis was conducted to examine this possibility. If the subjects were learning or adapting to the task, one would expect usefulness to increase throughout the study. Perceived usefulness should increase not only from categories to brands, but also across the three different stimulus sets used at each level. Another three-level order variable was added to the analysis-of-variance model to capture whether a particular set of brands or categories was presented first (fourth), second (fifth), or third (sixth) overall. This learning effect did not approach significance, and all other reported results remained unchanged. Thus, it is not easy to explain the observed difference between brands and categories on this basis.

Another possible limitation is that the authors used a group of analysts, in the form of students, who understood market analysis but were equally limited in their use of particular scaling techniques, It is important that future research relaxes this condition to explore the effects of managerial experience in this context. For example, managers who have more experience in the use of tree-based techniques may be in a better position to leverage their advantages. One solution is to explore the usefulness of trees and spaces in an environment that offers experimental control while allowing subjects to obtain more realistic experience, as in the case of market simulations (Glazer, Steckel, and Winer, 1988).

The primary implication of the research is that scaling techniques should be evaluated on objective statistical criteria as well as on user perceptions. A technique that provides marginally better fits to perceptual data may not be particularly useful in a market-analysis task. The purpose to which scaling techniques are put cannot be ignored. The perceived usefulness of alternative representations may even serve as a guide to developing new scaling techniques. Consider the observation that customer perceptions are better explained with the use of clustering schemes or feature trees, whereas managers find spatial solutions more useful. This suggests that spatial representations of feature-based or nonspatial perceptions should prove quite valuable. One example is the DeSarbo et al. (1992) TSCALE procedure, a spatial scaling technique which captures Tversky's (1977) feature-based model of perception with the use of abstract, latent dimensions. Overall the present study highlights the role of the user of market research methods. It is hoped that these results prove valuable to both the users and creators of such methods. 


\section{REFERENCES}

1. Alba, J. W., \& Hutchinson, J. W. (1987). Dimensions of consumer expertise. Journal of Consumer Research, 13, 411-454.

2. Anderson, J. R. (1983). A spreading activation theory of memory. Journal of Verbal Learning and Verbal Behavior, 22, 261-295.

3. Bauer, H. H., \& Hermann, A. (1995). Market demarcation: Theoretical framework and results of an empirical investigation of the German car market. European Journal of Marketing, 29(11), 18-34.

4. Carroll, J. D. (1976). Spatial, non-spatial and hybrid models for scaling. Psychometrika, 41, 439-463.

5. Corter, J. E. \& Tversky, A. (1986). Extended similarity trees. Psychometrika,

6. DeSarbo, W. S., Johnson, M. D., Manrai, A. K., Manrai, L. A., \& Edwards, E. A. (1992). TSCALE: A new multidimensional scaling procedure based on Tversky's contrast model. Psychometrika, 57, 43 $-69$.

7. DeSarbo, W. S., \& Rao, V. R. (1984). GENFOLD2: A set of models and algorithms for the GENeral UnFOLDing analysis of preference/dominance data. Journal of Classification, 1, 147- 186.

8. Doyle, P. (1971). Nonmetric multidimensional scaling: A user's guide. European Journal of Marketing, 7, 82-88.

9. Glazer, R., Steckel, J. H., \& Winer, R. S. (1988). The formation of key marketing variable expectations and their impact on firm performance: Some experimental evidence. Marketing Science, 8, 18- 34 .

10. Green, P. E. (1975). Marketing applications of MDS: Assessment and outlook. Journal of Marketing, 39, 24-31.

11. Green, P. E., \& Carmone, F. J., \& Smith, S. M. (1989). Multidimensional scaling: Concepts and applications. Boston, MA: Allyn and Bacon.

12. Hauser, J. R. (1986). Agendas and consumer choice. Journal of Marketing Research, 23, 199-212.

13. Herrmann, A. (1992). Produktwahlverhalten: Erlauterung and weioterentwicklung von modellen zur analyse des productwahlverhaltens aus marketingtheoretischer sicht. Stuttgart: Schaffer-Poeschel Verlag.

14. Hodgkinson, G. P., Padmore, J., \& Tomes, A. E. (1991). Mapping consumers' cognitive structures: A comparison of similarity trees with multidimensional scaling and cluster analysis. European Journal of Marketing, 25(7), 41-60.

15. Johnson, M. D. (1984). Consumer choice strategies for comparing noncomparable alternatives. Journal of Consumer Research, 11, 741-753.

16. Johnson, M. D., \& Fornell, C. (1987). The nature and methodological implications of the cognitive representation of products. Journal of Consumer Research, 14, 214-228.

17. Johnson, M. D., \& Horne, D. A. (1992). An examination of the validity of direct product perceptions. Psychology \& Marketing, 9, 22 1-235.

18. Johnson, M. D., Lehmann, D. R., Fornell C., \& Horne, D. R. (1992). Attribute abstraction, featuredimensionality, and the scaling of product similarities. International Journal of Research in Marketing, 9, 131 - 147. 
19. Johnson, S. C. (1967). Hierarchical clustering schemes. Psychometrika, 32, Kinnear, T. C., \& Taylor, J. R. (1996). Marketing research: An applied approach. New York: McGraw-Hill.

20. Kruskal, J. B. (1964). Multidimensional scaling by optimizing goodness of fit to a nonmetric hypothesis. Psychometrika, 29, 1-27.

21. Levitt, T. (1960). Marketing myopia. Harvard Business Review, Reprint No. 75507.

22. MacKay, D. B., \& Zinnes, J. L. (1986). A probabilistic model for the multidimensional scaling of proximity and preference data. Marketing Science, 5, 325-344.

23. OShaughnessy, J. (1984). Competitive marketing: A strategic approach. Boston, MA: Allen \& Unwin.

24. Pruzansky, S., Tversky, A., \& Carroll, J. D. (1982). Spatial versus tree representations of proximity data. Psychometrika, 47(1), 3 - 24.

25. Rosch, E. (1977). Human categorization. In N. Warren (Ed.), Studies in crosscultural Psychology (Volume 1, pp. 1-49). London: Academic Press.

26. Roskam, E., \& Lingoes, J. C. (1970). MINISSA-1: A FORTRAN IV (GI program for the smallest space analysis of square symmetric matrices. Behavioral Science, 15, 204-205.

27. Sattath, S., \& Tversky, A. (1977). Additive similarity trees. Psychometrika, 42, 319-345.

28. Shepard, R. N. (1962). The analysis of proximities: Multidimensional scaling with an unknown distance function, I and 11. Psychometrika, 27, 125-140 and 219-246.

29. Shepard, R. N. (1972). Introduction to Volume I. In R. Shepard, A. Romney, \& S. Nerlove (Eds.), Multidimensional scaling: Theory and applications in the behavioral sciences, Volume I: Theory (pp.1-20). New York: Seminar Press.

30. Shepard, R. N. (1980). Multidimensional scaling, tree fitting, and clustering. Science, 210, 390-398.

31. Shepard, R. N. (1981). Psychophysical complementarity. In M. Kubovy \& J. Pomerantz (Eds.), Perceptual organization (pp. 279-341). Hillsdale, NJ: Erlbaum.

32. Srivastava, R. K., Leone, R. P., \& Shocker, A. D. (1981). Market structure analysis: Hierarchical clustering of products based on substitution in use. Journal of Marketing, 45, 38-48.

33. Tversky, A. (1977). Features of similarity. Psychological Review, 84, 327-352.

34. Tversky, A., \& Hutchinson, J. W. (1986). Nearest neighbor analysis of psychological spaces. Psychological Review, 86, 542-573.

35. Tversky, A., \& Sattath, S. (1979). Preference trees. Psychological Review, 86, 542-573. 